\title{
Clinical and histopathologic analysis of gynecological cancer: a single institute experience over 7 years
}

\author{
Soo-Young Lee ${ }^{1}$, Eunbyeol Kim ${ }^{1}$, Hyo-Shin $\mathrm{Kim}^{1}$, Yu-Jin Koo ${ }^{2}$, Dae-Hyung Lee ${ }^{2}$ \\ ${ }^{1}$ Department of Obstetrics and Gynecology, Yeungnam University Hospital, Daegu, Korea \\ ${ }^{2}$ Department of Obstetrics and Gynecology, Yeungnam University College of Medicine, Daegu, Korea
}

Received: December 17, 2019

Revised: February 6, 2020

Accepted: February 18, 2020

Corresponding author:

Yu-Jin Koo

Department of Obstetrics and Gynecology, Yeungnam University

College of Medicine, 170

Hyeonchung-ro, Nam-gu, Daegu

42415, Korea

Tel: +82-53-620-3433

Fax: +82-53-654-0676

E-mail: yujinkoo@yu.ac.kr
Background: Approximately 100,000 women are diagnosed with cancer each year in Korea. According to a survey by the Korean central cancer registry in 2016, uterine cervical cancer, uterine corpus cancer, and ovarian cancer were the 5th, 7th, and 8th most prevalent cancers respectively among Korean women. The present study aims to review the clinico-pathologic characteristics of patients who were treated for major gynecological malignancies at Yeungnam University Medical Center.

Methods: Patients with invasive gynecological cancers from January 2012 to February 2019 were retrospectively identified. We analyzed the clinical features, demographic profiles, pathologic data, treatment modality used, adjuvant treatment used, complications, recurrence, and survival outcomes.

Results: A total of 287 patients (cervical cancer 115; corporal cancer 86; and ovarian, tubal, or primary peritoneal cancer 90 ) were included. Most cervical (82.7\%) and corporal cancers (89.5\%) were diagnosed in the early stages (stage I or II), while more than half (58.9\%) the cases of ovarian, tubal or peritoneal cancers were diagnosed in the advanced stages (stage III or IV). Surgical complications were observed in 12.2\% of cervical cancers, $16.3 \%$ of uterine corpus cancers, and $11.1 \%$ of ovarian, tubal, and peritoneal cancers, respectively. The 5 -year overall survival rate was $94.1 \%, 91.0 \%$, and $77.1 \%$ for cervical, corporal, and ovarian, tubal, or peritoneal cancers, respectively.

Conclusion: Surgical treatment was satisfactory in terms of the incidence of complications, and survival outcomes were generally good. Clinicians should be aware of the clinical and histopathological characteristics of patients with gynecological cancers to be able to provide optimal strategies and counseling.

Keywords: Endometrial neoplasms; Female genital neoplasms; Ovarian neoplasms; Uterine cervical neoplasms

\section{Introduction}

Cancer is a leading cause of morbidity and mortality, with approximately 14 million new cases diagnosed and 8 million cancer-related deaths worldwide in 2012 [1]. The three major gynecologic cancers are cervical, endometrial, and ovariancancer. Cervical cancer is the 4th most common malignancy in women worldwide and the disease resulted in over 300,000 deaths in 2018 [2]. Highrisk subtypes of the human papilloma virus (HPV) are the cause for most cervical cancers, and HPV screening and vaccination programs are effective for disease prevention. Endometrial cancer is the most common gynecologic malignancy in developed countries, with a rising incidence. In 2012, around 320,000 new cases of endometrial cancer were diagnosed worldwide [3]. Ovarian

Copyright (C) 2020 Yeungnam University College of Medicine

This is an Open Access article distributed under the terms of the Creative Commons Attribution Non-Commercial License (http://creativecommons.org/licenses/by-nc/4.0/) which permits unrestricted non-commercial use, distribution, and reproduction in any medium, provided the original work is properly cited. 
cancer is the common cause of gynecological cancer associated with death. One of the reasons for high mortality is late presentation in most cases. This cancer usually presents in postmenopausal women without typical symptoms. In 2018, about 295,000 new cases of ovarian cancer were diagnosed worldwide [4]. Based on the characteristics of gynecological cancers discussed above, the purpose of this study is to review the clinico-pathologic characteristics of patients with gynecological cancers treated at our hospital.

\section{Materials and methods}

We retrospectively identified patients with invasive gynecological cancers who were treated at Yeungnam University Medical Center from January 1, 2012 to February 28, 2019. This study was approved by the Institutional Review Board of the Yeungnam University Medical Center (IRB No: 2019-11-004). The disease codes used were according to the International Classification of Diseases, 10th edition, which included cervical cancer (C53), uterine corpus cancer (C54, C55), ovarian cancer (C56), tubal cancer (C57), and primary peritoneal cancer (C48). Cervical carcinoma in situ, borderline ovarian malignancy, vaginal cancer, and choriocarcinoma were excluded from the study. Patients who were transferred to other hospitals immediately after diagnosis were also excluded. Finally, 287 cases of gynecological cancer were included in this study. We analyzed the clinical features, demographic profiles, pathologic data, treatment modality used, adjuvant treatment used, complications, recurrence, progression-free survival (PFS), and overall survival (OS) of all patients. All cases were pathologically confirmed as gynecological cancer; however, a few cases of ovarian cancer were clinically diagnosed when cytological and imaging studies strongly suggested ovarian cancer. At our hospital, the baseline investigations for cervical cancer usually include serum squamous cell cancer-antigen (SCC-Ag) level, chest X-ray, cystoscopy, sigmoidoscopy, pelvic magnetic resonance imaging (MRI), and positron emission tomography-computed tomography (PET-CT). For endometrial cancer, the baseline investigations include serum cancer antigen-125 (CA-125) level, chest X-ray, sigmoidoscopy, pelvic MRI, and PET-CT. For ovarian cancer, the initial work-up includes the risk of ovarian malignancy algorithm (ROMA) test, chest X-ray, gastroscopy, colonoscopy, pelvic CT, and PET-CT. The stages of cervical and corporal cancer were classified according to the International Federation of Gynecology and Obstetrics (FIGO) classification 2009, and ovarian cancer was classified according to the FIGO stage 2014.

PFS was determined from the date of diagnosis to the date of first recurrence or the date of last follow-up. OS was determined from the date of diagnosis to the date of death or the date of last follow-up. Statistical analyses were performed using the IBM SPSS version 20.0 (IBM Corp., Armonk, NY, USA). Survival analyses were conducted using the Kaplan-Meier method, and surviving patients were censored at the date of last follow-up.

\section{Results}

\section{Cervical cancer}

Demographic characteristics and clinical features of patients with cervical cancer $(n=115)$ are described in Table 1 . The mean age at diagnosis was 53.8 years. The most common presenting symptom was vaginal bleeding, seen in 55 patients $(47.8 \%)$. Forty-seven patients (40.9\%) were diagnosed with cervical cancer during routine health screening. More than half of the patients (67.0\%) were diagnosed at stage I, while $15.7 \%$ were diagnosed at stage II. Serum SCC-Ag test was performed in 81 patients, and 43 of them (53.1\%) had abnormal results ( $>1.5 \mathrm{ng} / \mathrm{mL})$. The mean SCCAg level was $5.5 \mathrm{ng} / \mathrm{mL}$ at diagnosis. The most frequent histological type was squamous cell carcinoma (74.8\%). Adenocarcinomas and adenosquamous carcinomas were found in $18.3 \%$ and $1.7 \%$ of the cases respectively. Fifty-two patients (45.2\%) underwent only surgery while 26 patients $(22.6 \%)$ received concurrent chemoradiation therapy as front-line therapy. Most patients (96.2\%) treated with chemoradiotherapy received cisplatin alone. The most common surgical modality was open surgery ( 35 cases, $30.4 \%$ ), followed by laparoscopic surgery (31 cases, $27.0 \%$ ). Surgical complications occurred in 14 patients including bladder dysfunction in five patients, pelvic abscess in two patients, wound dehiscence in two patients, and deep vein thrombosis, sciatic neuropathy, vaginal vault bleeding, ureter injury and postoperative sepsis in one patient each. During the 32 months of median follow-up period, there were 17 cases with recurrence and seven deaths. Five-year PFS was $81.0 \%$, and 5-year OS was 94.1\%. Median PFS and OS were not reached. PFS and OS for each cancer site are shown in Fig. 1.

\section{Uterine corpus cancer}

Demographic characteristics and clinical features $(n=86)$ are described in Table 2. The mean age at diagnosis was 56.9 years. The most common presenting symptom was vaginal bleeding, in 66 patients (76.7\%). Nine patients (10.5\%) were diagnosed during routine health screening. Most cases $(83.7 \%)$ were in stage I at diagnosis and $9.3 \%$ of the cases were in stage III. Mean serum CA125 level was $59.5 \mathrm{U} / \mathrm{mL}$ at diagnosis. The most frequent histological type was endometrioid carcinoma $(69.8 \%)$, followed by serous carcinomas (7.0\%) and carcinosarcoma (7.0\%). Leiomyo- 
Table 1. Clinical and histopathologic characteristics of the uterine cervical cancers $(n=115)$

\begin{tabular}{|c|c|}
\hline Clinicopathologic characteristic & Value \\
\hline Age (yr) & $53.8(31-86)$ \\
\hline \multicolumn{2}{|l|}{ Symptom } \\
\hline Vaginal bleeding & $55(47.8)$ \\
\hline Incidentally detected on routine health screening & $47(40.9)$ \\
\hline Abdominal pain & $6(5.2)$ \\
\hline Vaginal discharge & $5(4.3)$ \\
\hline Others ${ }^{a}$ & $2(1.7)$ \\
\hline Gravidity (no. of times) & $4.0(0-30)$ \\
\hline Parity (no. of times) & $2.3(0-9)$ \\
\hline Body mass index $\left(\mathrm{kg} / \mathrm{m}^{2}\right)$ & $23.2(15-34)$ \\
\hline History of non-gynecologic malignancy & $11(9.6)$ \\
\hline Serum SCC-Ag at diagnosis (ng/mL) & $5.5(0.2-70)$ \\
\hline Tumor size $e^{b)}(\mathrm{cm})$ & $3.1(0.1-21)$ \\
\hline Synchronous primary cervical and endometrial cancers & $1(0.9)$ \\
\hline \multicolumn{2}{|l|}{ Histology type } \\
\hline Squamous cell carcinoma & $86(74.8)$ \\
\hline Adenocarcinoma & $21(18.3)$ \\
\hline Adenosquamous carcinoma & $2(1.7)$ \\
\hline Others ${ }^{c)}$ & $6(5.2)$ \\
\hline \multicolumn{2}{|l|}{ FIGO stage } \\
\hline I & $77(67.0)$ \\
\hline II & $18(15.7)$ \\
\hline III & $7(6.1)$ \\
\hline IV & $13(11.3)$ \\
\hline \multicolumn{2}{|l|}{ Initial treatment } \\
\hline Surgery alone & $52(45.2)$ \\
\hline Concurrent chemoradiation & $26(22.6)$ \\
\hline Surgery+radiotherapy & $11(9.6)$ \\
\hline Radiotherapy alone & $8(7.0)$ \\
\hline Surgery+concurrent chemoradiation & $7(6.1)$ \\
\hline Surgery+adjuvant chemotherapy & $4(3.5)$ \\
\hline $\begin{array}{l}\text { Neoadjuvant chemotherapy+surgery } \pm \text { any adjuvant } \\
\text { therapy }\end{array}$ & $5(4.3)$ \\
\hline Others & $2(1.7)$ \\
\hline \multicolumn{2}{|l|}{ Surgical mode } \\
\hline Abdominal & $35(30.4)$ \\
\hline Laparoscopic & $31(27.0)$ \\
\hline Robotic & $4(3.5)$ \\
\hline Vaginal (wide conization) & $12(10.4)$ \\
\hline \multicolumn{2}{|l|}{ Hysterectomy procedures } \\
\hline Extrafascial hysterectomy & $24(20.9)$ \\
\hline Modified radical hysterectomy & $9(7.8)$ \\
\hline Radical hysterectomy & $37(32.2)$ \\
\hline Pelvic lymphadenectomy & $50(43.5)$ \\
\hline Paraaortic lymphadenectomy & $21(18.3)$ \\
\hline Surgical complications & $14(12.2)$ \\
\hline Recurrence or progression & $17(14.8)$ \\
\hline Death & $7(6.1)$ \\
\hline
\end{tabular}

Values are presented as number (\%) or mean (range).

SCC-Ag, serum squamous cell cancer-antigen; FIGO, International Federation of Gynecology and Obstetrics.

a) Abdominal distension in one; headache in one case. ${ }^{b}$ Maximal diameter of the tumor either on the initial imaging study or on the pathologic finding. ${ }^{c)}$ Mucinous carcinoma in two; diffuse large B-cell lymphoma in one; neuroendocrine carcinoma in one; adenosarcoma in one; mixed carcinoma in one case.

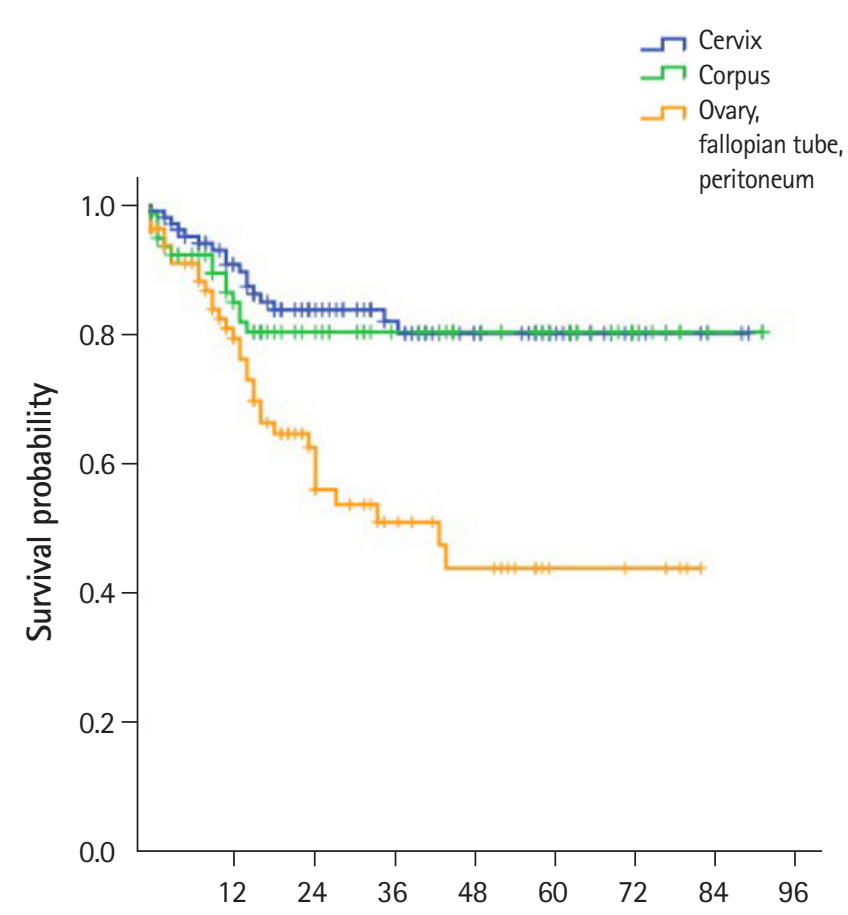

A

Time after diagnosis (mo)

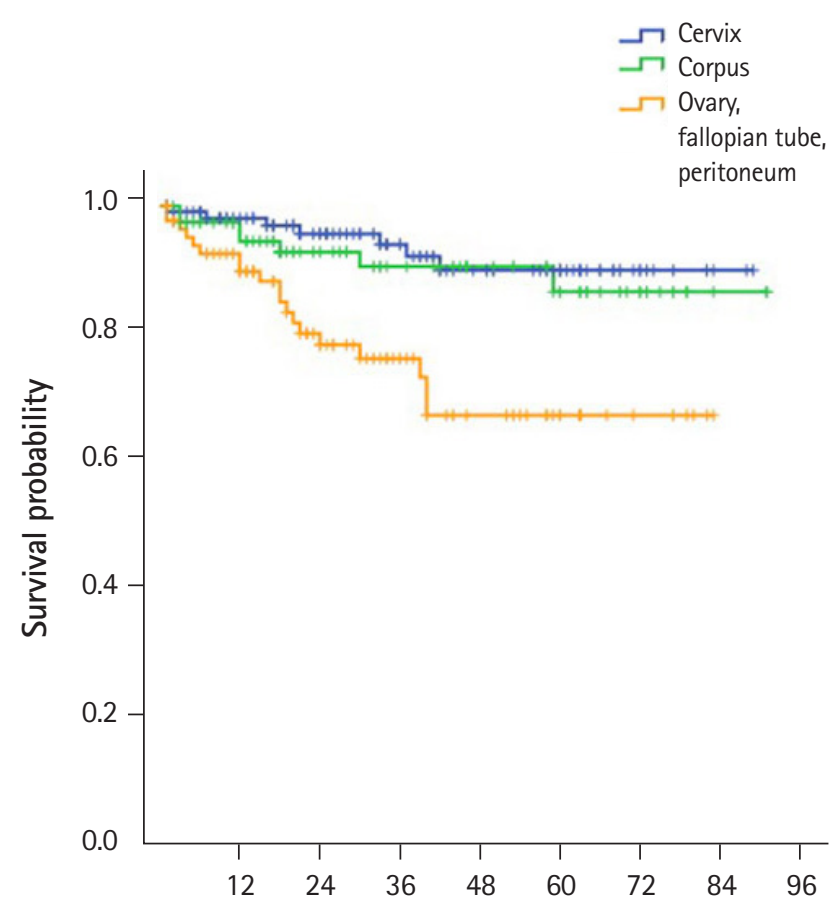

B

Time after diagnosis (mo)

Fig. 1. Progression-free survival (A) and overall survival (B) of the patients by the cancer sites. 
Table 2. Clinical and histopathologic characteristics of the uterine corpus cancers $(n=86)$

\begin{tabular}{|c|c|}
\hline Clinicopathologic characteristic & Value \\
\hline Age (yr) & $56.9(29-85)$ \\
\hline \multicolumn{2}{|l|}{ Symptom } \\
\hline Vaginal bleeding & $66(76.7)$ \\
\hline Incidentally detected on routine health screening & $9(10.5)$ \\
\hline Abdominal pain & $4(4.7)$ \\
\hline Abdominal distension & $4(4.7)$ \\
\hline Palpable pelvic mass & $3(3.5)$ \\
\hline Vaginal discharge & $2(2.3)$ \\
\hline Gravidity (no. of times) & $3.7(0-21)$ \\
\hline Parity (no. of times) & $2.1(0-7)$ \\
\hline Body mass index $\left(\mathrm{kg} / \mathrm{m}^{2}\right)$ & $25.3(17.1-38.9)$ \\
\hline History of non-gynecologic malignancy & $13(15.1)$ \\
\hline Serum CA-125 level at diagnosis $(\mathrm{U} / \mathrm{mL})$ & $59.5(1.1-1,220)$ \\
\hline Tumor size $^{\text {a) }}(\mathrm{cm})$ & $4.1(0.2-20)$ \\
\hline $\begin{array}{l}\text { Synchronous primary endometrial and ovarian can- } \\
\text { cers }\end{array}$ & $3(3.5)$ \\
\hline \multicolumn{2}{|l|}{ Histologic type } \\
\hline Endometrioid carcinoma & $60(69.8)$ \\
\hline Serous carcinoma & $6(7.0)$ \\
\hline Carcinosarcoma & $6(7.0)$ \\
\hline Leiomyosarcoma & $4(4.7)$ \\
\hline Endometrial stromal sarcoma & $2(2.3)$ \\
\hline Others ${ }^{b)}$ & $8(9.3)$ \\
\hline \multicolumn{2}{|l|}{ Histologic grade in case of endometrioid carcinoma } \\
\hline 1 & $30(34.9)$ \\
\hline 2 & $24(27.9)$ \\
\hline 3 & $6(7)$ \\
\hline \multicolumn{2}{|l|}{ FIGO stage } \\
\hline I & 72 (83.7) \\
\hline II & $5(5.8)$ \\
\hline III & $8(9.3)$ \\
\hline IV & $1(1.2)$ \\
\hline \multicolumn{2}{|l|}{ Initial treatment } \\
\hline Surgery alone & $45(52.3)$ \\
\hline Surgery+adjuvant chemotherapy & $21(24.4)$ \\
\hline Surgery+radiotherapy & $16(18.6)$ \\
\hline Surgery+chemoradiation & $2(2.3)$ \\
\hline Surgery+hormone therapy & $2(2.3)$ \\
\hline \multicolumn{2}{|l|}{ Surgical mode } \\
\hline Abdominal & $45(52.3)$ \\
\hline Laparoscopic & $36(41.9)$ \\
\hline Robotic & $4(4.7)$ \\
\hline Vaginal (dilatation and curettage) & $1(1.2)$ \\
\hline Pelvic lymphadenectomy & $53(61.6)$ \\
\hline Paraaortic lymphadenectomy & $36(41.9)$ \\
\hline Surgical complications & $14(16.3)$ \\
\hline Recurrence or progression & $16(18.6)$ \\
\hline Death & $7(8.1)$ \\
\hline
\end{tabular}

Values are presented as numbers (\%) or means (range). CA-125, cancer antigen-125; FIGO, International Federation of Gynecology and Obstetrics.

${ }^{a)}$ Maximal diameter of the tumor either on the initial imaging study or on the pathologic finding. ${ }^{b}$ Mixed carcinoma in four; mucinous carcinoma in two; undifferentiated carcinoma in one; Mullerian adenosarcoma in one. sarcoma and endometrial stromal sarcoma were diagnosed in $4.7 \%$ and $2.3 \%$ of the cases respectively. Forty-five patients (52.3\%) underwent only surgery. A case in stage IA underwent dilatation and curettage followed by adjuvant hormonal therapy using levonorgestrel-releasing intrauterine device for preservation of fertility. Following surgery, 21 patients $(24.4 \%)$ received adjuvant chemotherapy, 16 patients $(18.6 \%)$ received radiotherapy and two patients $(2.3 \%)$ received adjuvant chemoradiation. Almost half of the patients (47.6\%) were treated with adjuvant chemotherapy using paclitaxel and carboplatin, and the rest with cisplatin-based combined regimen. The most common surgical modality was open surgery ( 45 cases, $52.3 \%$ ), followed by laparoscopic surgery (36 cases, $41.9 \%$ ). The following surgical complications were observed: wound dehiscence in six patients, infection at the drainage site in two patients, and ureteral injury, bladder injury, vaginal wall laceration, small bowel injury, voiding difficulty and intracranial hemorrhage in one patient each. During the 28 months of median follow-up, there were 16 cases with recurrence and seven deaths. The 5-year PFS was 79.3\%, and 5-year OS was 91.0\%. Median PFS and OS were not reached.

\section{Ovarian, tubal and primary peritoneal cancer}

Pathologic characteristics and clinical features of patients with ovarian, tubal and peritoneal cancer $(n=90)$ are described in Table 3. The mean age was 56.3 years. The predominant presenting symptoms were abdominal pain (34.4\%), abdominal distension (22.2\%), vaginal bleeding (12.2\%), and palpable pelvic mass (10.0\%). Cases of incidental diagnosis were $14.4 \%$. Serum CA-125 test was conducted in 83 out of 90 patients, and 60 of them (72.3\%) had abnormal results ( $>35 \mathrm{U} / \mathrm{mL}$ ). The mean CA-125 level was $727.6 \mathrm{U} / \mathrm{mL}$ at diagnosis. In our hospital, Breast Cancer Susceptibility Gene (BRCA) mutation test was initiated at the department of gynecology in February 2016 on a whole blood sample using polymerase chain reaction and direct sequencing. In all, 18 patients with ovarian, tubal, or peritoneal cancer underwent the test, and $4(22.2 \%)$ were positive: BRCA1 and BRCA2 were positive in two patients each. The site of primary cancer was ovaries in $87.8 \%$, fallopian tubes in $3.3 \%$, and peritoneum in $5.6 \%$. More than half the patients $(58.9 \%)$ were in the advanced stage (stage III or IV) at diagnosis. The most common epithelial histological type was serous adenocarcinoma (53.3\%) while mucinous carcinoma and clear cell carcinoma were found in $10.0 \%$ each. Twenty patients $(22.2 \%)$ underwent only surgery, while 54 patients $(60.0 \%)$ received postoperative adjuvant chemotherapy. Ten patients (11.1\%) underwent surgery after neoadjuvant chemotherapy. Paclitaxel plus carboplatin was used in $90.0 \%$ of the neoadjuvant chemotherapy regimens and cyclophosphamide plus cispla- 
Table 3. Clinical and histopathologic characteristics of the ovarian, tubal, and primary peritoneal cancers $(n=90)$

\begin{tabular}{|c|c|}
\hline Clinicopathologic characteristic & Value \\
\hline Age (yr) & $56.3(13-80)$ \\
\hline \multicolumn{2}{|l|}{ Symptom } \\
\hline Abdominal pain & $31(34.4)$ \\
\hline Abdominal distension & $20(22.2)$ \\
\hline Incidentally detected on routine health screening & $13(14.4)$ \\
\hline Vaginal bleeding & $11(12.2)$ \\
\hline Palpable pelvic mass & $9(10.0)$ \\
\hline Others & $7(7.8)$ \\
\hline Gravidity (no. of times) & $2.8(0-8)$ \\
\hline Parity (no. of times) & $1.7(0-6)$ \\
\hline Body mass index $\left(\mathrm{kg} / \mathrm{m}^{2}\right)$ & $22.8(15.9-30)$ \\
\hline History of non-gynecologic malignancy & $13(14.4)$ \\
\hline Serum CA-125 level at diagnosis (U/mL) & $727.6(0.1-7,187.9)$ \\
\hline Tumor size $^{a}(\mathrm{~cm})$ & $9.6(0.5-25)$ \\
\hline \multicolumn{2}{|l|}{ Primary cancer site } \\
\hline Ovary & 79 (87.8) \\
\hline Synchronous primary endometrial and ovarian cancers & $3(3.3)$ \\
\hline Fallopian tube & $3(3.3)$ \\
\hline Peritoneum & $5(5.6)$ \\
\hline \multicolumn{2}{|l|}{ Histologic type } \\
\hline Serous & $48(53.3)$ \\
\hline Mucinous & $9(10.0)$ \\
\hline Clear cell & $9(10.0)$ \\
\hline Endometrioid & $8(8.9)$ \\
\hline Others ${ }^{b)}$ & $10(11.1)$ \\
\hline Unknown ${ }^{c)}$ & $6(6.7)$ \\
\hline \multicolumn{2}{|l|}{ Histologic grade in case of serous carcinoma } \\
\hline Low-grade & $2(2.2)$ \\
\hline High-grade & $45(50)$ \\
\hline Unknown & $1(1.1)$ \\
\hline
\end{tabular}

\begin{tabular}{|c|c|}
\hline Clinicopathologic characteristic & Value \\
\hline \multicolumn{2}{|l|}{ FIGO stage } \\
\hline I & $33(36.7)$ \\
\hline ॥ & $4(4.4)$ \\
\hline III & $34(37.8)$ \\
\hline IV & $19(21.1)$ \\
\hline \multicolumn{2}{|l|}{ Initial treatment } \\
\hline Surgery alone & $20(22.2)$ \\
\hline Surgery+adjuvant chemotherapy & $54(60.0)$ \\
\hline $\begin{array}{l}\text { Neoadjuvant chemotherapy+surgery+adjuvant } \\
\text { chemotherapy }\end{array}$ & $9(10.0)$ \\
\hline Neoadjuvant chemotherapy+surgery & $1(1.1)$ \\
\hline Palliative chemotherapy & $5(5.6)$ \\
\hline Supportive care alone & $1(1.1)$ \\
\hline \multicolumn{2}{|l|}{ Surgical mode } \\
\hline Abdominal & $67(74.4)$ \\
\hline Laparoscopic & $16(17.8)$ \\
\hline Robotic & $1(1.1)$ \\
\hline \multicolumn{2}{|l|}{ Fertility-sparing surgery } \\
\hline Unilateral salpingo-oophorectomy & $4(4.4)$ \\
\hline Ovarian cystectomy & $1(1.1)$ \\
\hline \multicolumn{2}{|l|}{ Postoperative residual tumor } \\
\hline Grossly none & $52(57.8)$ \\
\hline Size $<2 \mathrm{~cm}$ & $9(10.0)$ \\
\hline Size $\geq 2 \mathrm{~cm}$ & $23(25.6)$ \\
\hline Pelvic lymphadenectomy & $49(54.4)$ \\
\hline Paraaortic lymphadenectomy & $43(47.8)$ \\
\hline Surgical complications & $10(11.1)$ \\
\hline Recurrence or progression & $34(37.8)$ \\
\hline Death & $19(21.1)$ \\
\hline
\end{tabular}

Values are presented as numbers (\%) or means (range).

CA-125, cancer antigen-125; FIG0, International Federation of Gynecology and Obstetrics.

${ }^{\text {a) }}$ Maximal diameter of the tumor either on the initial imaging study or on the pathologic finding. ${ }^{\text {b) }}$ Adenocarcinoma in two; immature teratoma in two; adult granulosa cell tumor in one; transitional cell carcinoma in one; small cell carcinoma in one; undifferentiated carcinoma in one; dysgerminoma in one; poorly differentiated carcinoma in one case. ${ }^{c}$ Clinically diagnosed by both ascites cytology and imaging studies.

tin was used in the remaining $10.0 \%$. Bevacizumab has been introduced in January 2015 in our hospital and has been used in 11 patients. The most common surgical modality was open surgery (67 cases, $74.4 \%$ ), followed by laparoscopic surgery (16 cases, $17.8 \%)$. Five patients (5.5\%) underwent fertility preserving surgery. The following surgical complications were observed: wound dehiscence in five patients, and bowel leak, bladder injury, deep vein thrombosis, acute kidney injury and cerebral infarction in one patient each. During the 23 months of median follow-up, there were 34 recurrences and 19 deaths. The 5-year PFS was $62.0 \%$, and 5-year OS was 77.1\%. Median PFS was 43 months (95\% confi- dence interval, 20.5-65.5), and median OS was not reached.

\section{Discussion}

Cervical cancer is the most common gynecological cancer in developing countries while endometrial cancer is the most common gynecological cancer in developed countries. The incidence of ovarian cancer is also higher in developed countries than in developing countries. In line with this trend, the incidence of cervical cancer is gradually decreasing, while that of uterine corporal and ovarian cancers is increasing in Korea. Our data showed the over- 
all clinico-pathologic results in patients who were treated for major gynecological cancers at our hospital. Limitations of the present study include its retrospective design, small sample size, and single-group analysis without comparison of data from other institutions. Nevertheless, this review of our data presents an opportunity to understand the characteristics of local population with gynecological malignancies and to evaluate the outcomes of our management.

The incidence and mortality of cervical cancer have decreased since the introduction of screening programs. Furthermore, in countries where HPV vaccination program has been introduced, a substantial decrease in the incidence of cervical cancer is expected. According to the 2015 annual report of cancer statistics in Korea, the incidence of cervical cancer was high in the age groups of 40s, 50s and 30s [5]. Similarly, our data showed that the incidence was highest with $29.6 \%$ in the 50 s, followed by $21.7 \%$ in the 40 s, and $17.4 \%$ in the 30s. The Surveillance, Epidemiology, and End Results (SEER) data in the United States reported the incidence of cervical cancer diagnosed in different stages as follows: $44 \%$ for localized disease, $36 \%$ for regional disease, and $15 \%$ for distant disease [6]. The incidence of different stages of cancer in our data was slightly different: $67.0 \%$ localized disease, $21.7 \%$ regional disease, and $11.3 \%$ distant disease. Such a high proportion of localized disease seems to be due to active national cervical cancer screening. Globally, squamous cell carcinoma and adenocarcinoma are the most common histological subtypes accounting for approximately $70 \%$ and $25 \%$ of all cervical cancers, respectively [2]. The histological analysis of cervical cancer patients in our hospital showed $74.8 \%$ to be squamous cell carcinoma, $18.3 \%$ adenocarcinoma, and $1.7 \%$ adenosquamous cell carcinoma, which is in line with the global trend. According to the SEER data, the 5-year survival rate was $65.5 \%$ in 2015 [6], while the 5-year survival rate in the patients in our study was $94.1 \%$. There might be various factors related to the difference in survival rates such as ethnic, racial, sociocultural, and other demographic characteristics, as well as the national medical policy and program. In addition, a better survival rate could be partially because our study included more cases in the early stage of the disease as follows: 25 cases $(21.7 \%)$ in stage IA1, 5 cases $(4.3 \%)$ in IA2, 36 cases $(31.3 \%)$ in stage IB1, and 11 cases $(9.6 \%)$ in stage IB2. The surgical treatment appeared to be generally tolerable in terms of the incidence (12.2\%) and severity of complications.

In endometrial cancer, the most common histologic type is endometrioid adenocarcinoma (80\%), while the other types are mucinous carcinoma (5\%), papillary serous carcinoma (3\%-4\%), and clear cell carcinoma $(<5 \%)[7]$. The standard treatment for early-stage endometrial cancer is surgery including total hysterec- tomy and bilateral salpingo-oophorectomy. The safety and advantages of laparoscopic surgery have been proven in several randomized clinical trials $[8,9]$. In our data, the rate of laparoscopic surgery was quite high (41.8\%). According to the SEER data, the 5 -year survival rate was $81.0 \%$ in 2015 , while the 5-year survival rate in the patients in our study was $91.0 \%$ [10].

BRCA $1 / 2$ mutations are the most frequently identified genetic changes associated with ovarian cancer. It has been recently known that a BRCA $1 / 2$ germline mutation is associated with poor prognostic factors such as early-onset cancer, serous histology, and advanced stages of cancer [11]. The incidence of BRCA $1 / 2$ mutations has been reported to be $16.5 \%$ in the ovarian cancer patients in Korea [12]. In our data, BRCA $1 / 2$ mutation was seen in $22.2 \%$ of the patients with ovarian cancer, which is probably due to the small sample size in the present study. In addition, clear cell carcinoma was found in a high proportion $(10.0 \%)$ of ovarian cancer patients. In general, clear cell carcinoma accounts for about $5 \%$ of all ovarian cancers [13]. According to the SEER data, the 5-year survival rate was $47.8 \%$ in 2015 , while the 5-year survival rate in the patients in our study was 77.1\% [14]. More cases detected at an early stage might be a reason for the better survival. The distribution of various stages of cancer was as follows: localized malignancy and distant metastasis $15 \%$ and 59\% respectively in the SEER data and $36.7 \%$ and $21.1 \%$ respectively in our data.

In summary, most characteristics were similar with those of the worldwide data. The survival outcomes of patients with gynecological cancer in our data were generally good. Clinicians should well be aware of the clinical and histopathological characteristics of the patients with gynecological cancer to be able to provide optimal management options.

\section{Acknowledgments}

\section{Conflicts of interest}

No potential conflict of interest relevant to this article was reported.

\section{Author contributions}

Conceptualization: YJK; Data curation: SYL, EK, HSK; Formal analysis: SYL, YJK; Methodology: YJK; Project administration: DHL; Software: YJK; Supervision: YJK, DHL; Writing-original draft: SYL; Writing-review \& editing: YJK.

\section{ORCID}

Soo-Young Lee, https://orcid.org/0000-0001-8672-0751 Eunbyeol Kim, https://orcid.org/0000-0002-4345-637X Hyo-Shin Kim, https://orcid.org/0000-0002-8369-417X 
Yu-Jin Koo, https://orcid.org/0000-0002-0219-0317

Dae-Hyung Lee, https://orcid.org/0000-0002-5114-8000

\section{References}

1. Forman D, Ferlay J. The global and regional burden of cancer. In: Stewart BW, Wild CP, editors. World cancer report 2014. Lyon (FR): International Agency for Research on Cancer; 2014. p. 16-53.

2. Cohen PA, Jhingran A, Oaknin A, Denny L. Cervical cancer. Lancet 2019;393:169-82.

3. Morice P, Leary A, Creutzberg C, Abu-Rustum N, Darai E. Endometrial cancer. Lancet 2016;387:1094-108.

4. International Agency for Research on Cancer. Global cancer observatory: cancer today [Internet]. Lyon (FR): International Agency for Research on Cancer; 2018 [cited 2020 Jan 22]. http://gco.iarc.fr/.

5. Korea Central Cancer Registry, National Cancer Center. Annual report of cancer statistics in Korea in 2015. Sejong (KR): Ministry of Health and Welfare; 2017.

6. Howlader N, Noone AM, Krapcho M, Miller D, Brest A, Yu M, et al. SEER cancer statistics review, 1975-2016: cancer of the cervix uteri (invasive) [Internet]. Bethesda (MD): National Cancer Institute; 2019 [cited 2019 Sep 25]. https://seer.cancer.gov/ csr/1975_2016/results_merged/sect_05_cervix_uteri.pdf.

7. Dowdy SC, Mariani A, Lurain JR. Uterine cancer. In: Berek JS, editor. Berek \& Novak's gynecology. 15th ed. Philadelphia (PA): Lippincott Williams \& Wilkins; 2012.p. 1250-303.

8. Janda M, Gebski V, Brand A, Hogg R, Jobling TW, Land R, et al. Quality of life after total laparoscopic hysterectomy versus total abdominal hysterectomy for stage I endometrial cancer (LACE): a randomised trial. Lancet Oncol 2010;11:772-80.

9. Lu Q, Liu H, Liu C, Wang S, Li S, Guo S, et al. Comparison of laparoscopy and laparotomy for management of endometrial carcinoma: a prospective randomized study with 11-year experience.J Cancer Res Clin Oncol 2013;139:1853-9.

10. Howlader N, Noone AM, Krapcho M, Miller D, Brest A, Yu M, et al. SEER cancer statistics review, 1975-2016: cancer of the corpus and uterus, NOS (invasive) [Internet]. Bethesda (MD): National Cancer Institute; 2019 [cited 2019 Sep 25]. https:// seer.cancer.gov/csr/1975_2016/results_merged/sect_07_ corpus_uteri.pdf.

11. Lakhani SR, Manek S, Penault-Llorca F, Flanagan A, Arnout L, Merrett S, et al. Pathology of ovarian cancers in BRCA1 and BRCA2 carriers. Clin Cancer Res 2004;10:2473-81.

12. Kwon BS, Byun JM, Lee HJ, Jeong DH, Lee TH, Shin KH, et al. Clinical and genetic characteristics of BRCA1/2 mutation in Korean ovarian cancer patients: a multicenter study and literature review. Cancer Res Treat 2019;51:941-50.

13. Berek JS, Longacre TA, Friedlander M. Ovarian, fallopian tube, and peritoneal cancer. In: Berek JS, editor. Berek \& Novak's gynecology. 15th ed. Philadelphia: Lippincott Williams \& Wilkins; 2012.p. 1350-427.

14. Howlader N, Noone AM, Krapcho M, Miller D, Brest A, Yu M, et al. SEER cancer statistics review, 1975-2016: cancer of the ovary (invasive) [Internet]. Bethesda (MD): National Cancer Institute; 2019 [cited 2019 Sep 25]. https://seer.cancer.gov/ csr/1975_2016/results_merged/sect_21_ovary.pdf. 